\title{
Giant superior mediastinal angioleiomyoma
}

\author{
Marian Vrtik, MBBS, ${ }^{a}$ Robert I. Larbalestier, MBBS, FRACS, ${ }^{a}$ David Cameron, MBBS, FRANZCR, ${ }^{\text {b }}$ \\ Ashu Gupta, BMedSC, MBBS, ${ }^{\mathrm{b}}$ and Rajalingam Sinniah, DSc, FRCPath, FRCPA, ${ }^{\mathrm{c}}$ Perth, Australia
}

W e report a case of a large, symptomatic angioleiomyoma originating from the right superior mediastinum with clinical and radiographic features suggestive of an invasive malignant process that was initially thought to be unsuitable for curative resection. The tumor was successfully resected through a right posterolateral thoracotomy, with an excellent postoperative recovery.

\section{Clinical Summary}

A 37-year-old white woman presented in May 2003 with a short history of productive cough and mild hemoptysis that failed to settle after an episode of upper respiratory tract infection. Her other symptoms included shortness of breath on moderate exertion, tiredness, and loss of appetite with minimal weight loss. Clinical examination was unremarkable, except for dullness to percussion and reduced breath sounds in the right upper zone. There were no abnormalities noted on both biochemical and hematologic analyses of the patient's blood.

Chest radiography showed a large opacity in the right upper zone (Figure 1). A computed tomographic scan-guided core biopsy, reported as smooth muscle tumor, could not exclude malignancy. A magnetic resonance imaging study revealed a right intrathoracic tumor with a well-defined thin capsule with no demonstrable tissue planes between the tumor and esophagus, the posterior membrane of distal trachea, and the right main bronchus (Figure 2). There was no obvious invasion of the lung, heart, and major mediastinal vessels.

On the basis of the clinical features, size, and radiographic appearance, the tumor was considered likely to be malignant and unresectable. After discussion with the patient and her oncologist, a debulking procedure was planned to obtain more pathologic material, to control symptoms, and to maximize the tumor response to adjuvant therapy.

A large $(21 \times 13 \times 11 \mathrm{~cm})$, apparently encapsulated, multilobulated mass was exposed (Figure 3, A) through the right pos-

From the Departments of Cardiothoracic Surgery, ${ }^{\mathrm{a}}$ Radiology, ${ }^{\mathrm{b}}$ and Anatomical Pathology, ${ }^{\mathrm{c}}$ Royal Perth Hospital, Perth, Australia.

Received for publication Feb 22, 2004; accepted for publication March 10, 2004.

Address for reprints: Marian Vrtik, MBBS, Department of Cardiothoracic Surgery, Royal Perth Hospital, Wellington Street Campus, Box X2213 GPO, Perth 6847, Western Australia, Australia (E-mail: maros96@ hotmail.com)

J Thorac Cardiovasc Surg 2004;128:786-8

$0022-5223 / \$ 30.00$

Copyright $\odot 2004$ by The American Association for Thoracic Surgery

doi:10.1016/j.jtcvs.2004.03.035 terolateral thoracotomy. The tumor was adherent to the upper mediastinum, middle esophagus, and both the right upper and middle lobes. Significant bleeding was encountered on attempting to debulk the tumor, and therefore we decided to attempt radical excision. A linear stapling device was used to separate the tumor from the lung. Surgical planes were developed between the tumor and mediastinal structures. Multiple feeding vessels were divided, and resection was successfully completed without any visible tumor left in situ. The patient had an uneventful recovery and was discharged on the seventh postoperative day.

Pathologic examination revealed a tumor with a cream-colored cut surface that was whorled in appearance, with multiple foci of softening but no areas of hemorrhage. It was composed of bundles of spindle-shaped smooth muscle cells forming intersecting fascicles. The cells seemed to originate from medium-sized blood vessels and were arranged circumferentially, radiating out from the outer layer of the blood vessels (Figure 3, B). There was no nuclear pleomorphism, and only one mitosis was noted in 50 high-power fields (magnification $40 \times$ ). In one focus there was pleural involvement, but no infiltration of the lung parenchyma was noted (Fig 3, $B$, inset). The tumor cells were positive for smooth muscle antigens. A diagnosis of a benign angioleiomyoma with pleural involvement was made.

\section{Discussion}

Mesenchymal tumors comprise a significant proportion of mediastinal lesions. ${ }^{1}$ However, to date, there have been only 2 cases of angioleiomyoma involving the deep structures within the chest reported in the literature. ${ }^{2,3}$ Angioleiomyoma is a rare and benign vascular tumor that more commonly develops in women and characteristically presents as a small-to-medium, painful, cutaneous or subcutaneous nodule in the extremities, head and neck region, and, on rarer occasions, in the trunk. ${ }^{4}$ Involvement of deep structures is extremely rare. Surgical excision is the treatment of choice, with a very low local recurrence rates. ${ }^{4}$

In our patient, on the basis of the clinical presentation, lack of tissue planes between the tumor, and the mediastinal structures on preoperative imaging, coupled with inconclusive biopsy results, the tumor was presumed to be an inoperable sarcoma rather than a benign growth. Unexpected brisk bleeding encountered while attempting to debulk the tumor forced us to perform radical resection. Both the size and vascular nature of the tumor made dissection very precarious. On the other hand, a macroscopically well-defined tumor capsule helped us separate the tumor from the mediastinal structures, with no visible tumor left in situ. Follow-up chest computed tomographic scanning 3 months after the operation showed no evidence of tumor recurrence. However, pleural involvement on microscopic examination might lead to local recurrence that might necessitate re-excision at some point in the future. $^{2}$ 

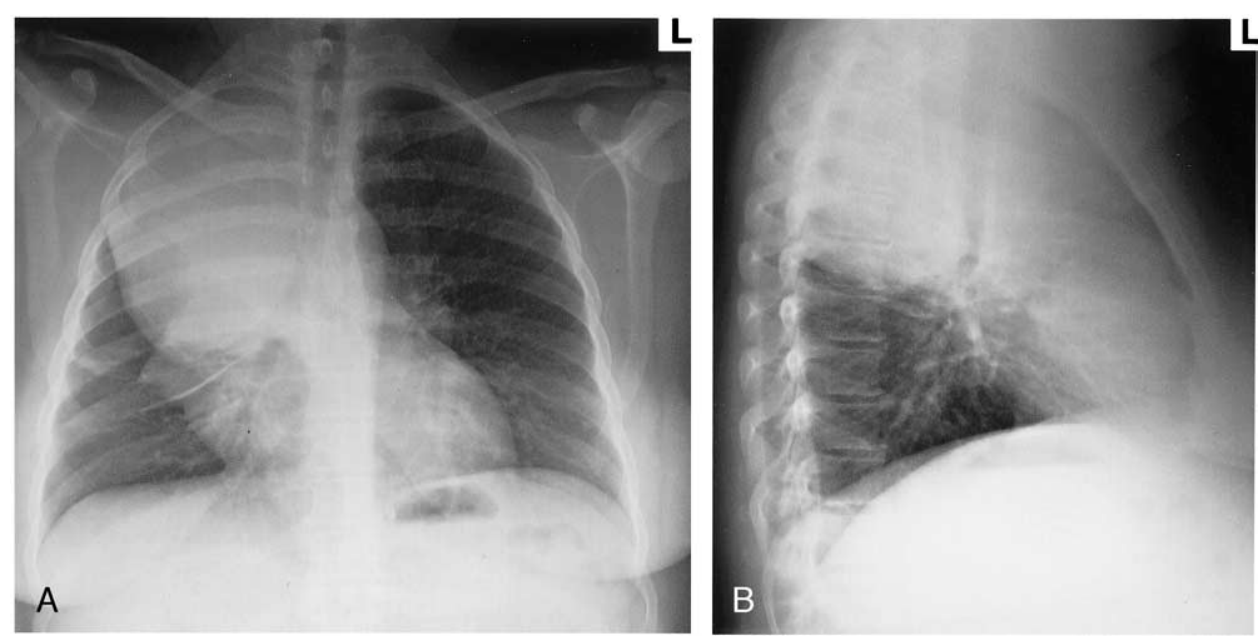

Figure 1. Posteroanterior (A) and lateral (B) chest radiographs reveal a large soft tissue mass occupying the superior right hemithorax.
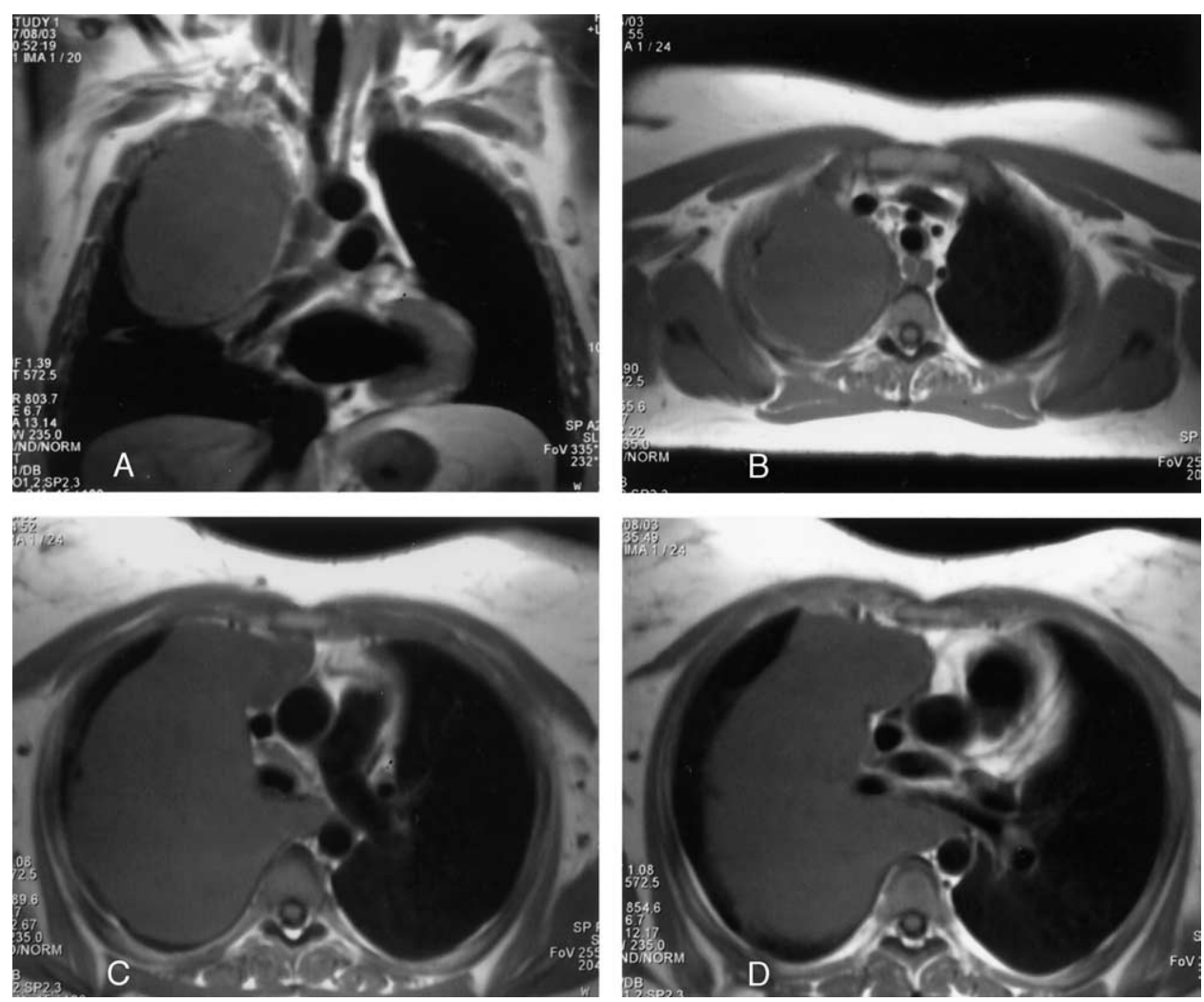

Figure 2. Magnetic resonance imaging scan with $\mathrm{T} 1$ weighting. The coronal scan (A) reveals that the soft tissue mass is almost completely encapsulated by tissue planes. This includes a fat plane separating it from superior mediastinal structures (B), which is an axial image at the level of the left brachiocephalic vein. However, tissue planes were not evident between the tumor and the distal trachea, right main bronchus, and middle third of the esophagus. This is shown in C, which is an axial image at the level of the carina, and D, at the level of the right main bronchus. 

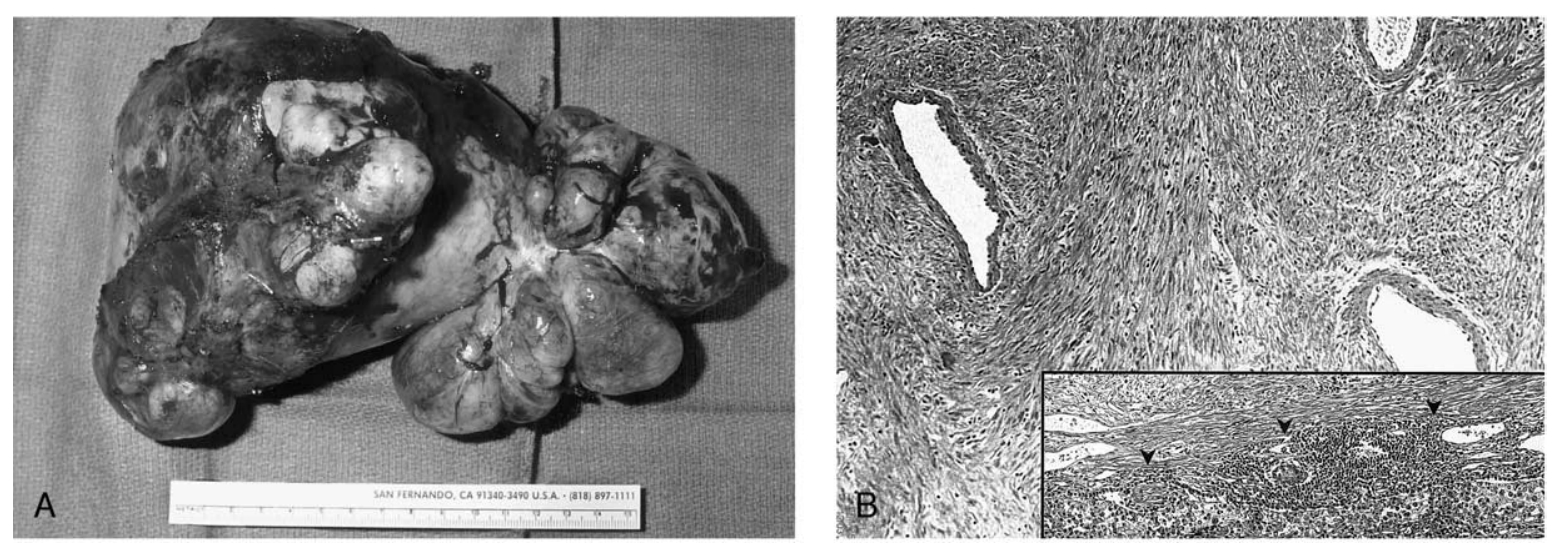

Figure 3. A, A large $(210 \times 130 \times 110 \mathrm{~mm})$, multilobulated, and apparently encapsulated tumor is shown. B, The tumor is composed of fascicles or bundles of spindle-shaped cells that originate from blood vessels. There is a focus of pleural involvement (inset) but no infiltration of lung parenchyma (arrowheads). (Original magnification 230x; hematoxylin and eosin stain.)

\section{References}

1. Davis RD Jr, Oldham HN Jr, Sabiston DC Jr. Primary cysts and neoplasms of the mediastinum: recent changes in clinical presentation, methods of diagnosis, management, and results. Ann Thorac Surg. 1987;44:229-37.
2. van Rijn ABB, van Kralingen KW, Koelma IA. Angioleiomyoma of the diaphragm. Ann Thorac Surg. 2000;69:1928-9.

3. Matsuoka H, Nishio W, Sakamoto T, Harada H, Sashikata T, Tsubota N. Mediastinal angioleiomyoma. Ann Thorac Surg. 2002;73:1653-4.

4. Hachisuga T, Hashimoto H, Enjoji M. Angioleiomyoma. A clinicopathologic reappraisal of 562 Cases. Cancer. 1984;54:126-30. 